Research Article

\title{
Boundary Effect and Dynamic Response Study for the Penetration of Rigid Projectiles into Thick, Finite-Radius, Metallic Targets
}

\author{
Juan Wang $\left(\mathbb{1},{ }^{1}\right.$ Junhai Zhao $\mathbb{D}^{2},{ }^{2}$ Yuan Zhou $\mathbb{1}^{1},{ }^{1}$ and Shanshan Sun $\mathbb{1}^{2}$ \\ ${ }^{1}$ College of Science, Chang'an University, Xi'an 710064, China \\ ${ }^{2}$ School of Civil Engineering, Chang'an University, Xi'an 710061, China
}

Correspondence should be addressed to Juan Wang; wangjuanhao@chd.edu.cn

Received 31 July 2020; Accepted 24 October 2020; Published 12 November 2020

Academic Editor: Zhixiong Li

Copyright (c) 2020 Juan Wang et al. This is an open access article distributed under the Creative Commons Attribution License, which permits unrestricted use, distribution, and reproduction in any medium, provided the original work is properly cited.

\begin{abstract}
This study uses the unified strength theory to analyse the elastoplastic stage and plastic stage of a linear strain-hardening target material while considering the effects of the intermediate principal stress and the free lateral boundaries of the target. In this investigation, analytical solutions of the radial stress in the cavity wall are obtained, and a unified penetration model of the target material is built. On this basis, penetration resistance formulas and penetration depth formulas for rigid projectiles with various nose shapes penetrating into thick, finite-radius, metallic targets are deduced, the solutions of which are obtained by utilizing the Simpson method. Accordingly, the proposed method offers a broader scope of application and higher accuracy than previous methods. Through this method, a series of analytical solutions based on different criteria can be obtained, and the penetration depth ranges of targets under different striking velocities can be effectively predicted. Moreover, penetration processes under different conditions are numerically simulated using the software ANSYS/LS-DYNA to study the motion law of the projectiles and the dynamic response of the targets. From the theoretical and numerical approaches, a list of influencing factors for terminal ballistic effects are analysed, including the strength criterion differences, the strength parameter $b$, the striking velocity $v_{0}$, the projectile nose shape, and the target radius-to-projectile radius ratio $r_{t} / a$. The results indicate that, as $b$ changes from 1 to 0 , the penetration depth $D_{\max }$ increases by $22.45 \%$. Additionally, $D_{\max }$ increases by $40.76 \%$ when $r_{t} / a$ changes from 16 to 4 ; hence, it cannot be calculated as an unlimited-size target anymore when $r_{t} / a \leq 16$. In weapons field tests, the radius of the metallic target can be conservatively designed to be greater than 28 times the projectile radius to ignore the effect from the free lateral boundaries of the target.
\end{abstract}

\section{Introduction}

The cavity expansion theory which has been widely used in the field of terminal ballistics can be sorted into two theories according to the different hypothesized cavity expansion styles: spherical cavity expansion theory and cylindrical cavity expansion theory. By applying these two theories respectively, investigators have studied penetration phenomena in several materials, such as metals, rocks, concrete, and soil, and many results have been obtained [1-10]. However, most studies on penetration have focused on semiinfinite targets or the finite-thickness targets (considering the free-surface on the back of the target), and systematic studies on penetration into thick, finite-radius, targets have been limited to experiments $[11,12]$. The few theoretical studies on penetration mechanics in such targets have generally ignored the effects of the lateral boundaries, and the experiments conducted by Littlefield [11] have shown that this assumption makes the results obviously deviate from reality when the in-plane dimensions of the target are small; that is, the ballistic performance of certain targets is known to be sensitive to confinement. On the other hand, the armoured targets encountered in practical applications and the real targets in experiments often have finite dimensions; hence, edge effects are bound to exist. Accordingly, the finite cylindrical cavity expansion theory was 
proposed by Jiang et al. [13] in 2011; moreover, they conducted the first systematic investigation on the penetration of long-rod projectiles into thick metal targets with finite inplane dimensions. Then, an engineering model for the penetration of rigid sharp-nosed projectiles into thick, finiteradius, metal targets was presented by Song et al. [14]. However, this model adopting the von Mises yield criterion is imperfect and can only be used for projectiles with a single nose shape and for materials with an ultimate shear strength that is 0.577 times its yield strength in tension or compression. Even if adopting the Tresca yield criterion or Twin shear stress yield criterion, this model is only applied for materials with an ultimate shear strength that is 0.5 or 0.667 times its yield strength in tension or compression, respectively. Hence, the application scope of this model is limited.

Since the unified strength theory, which considers all the stress components acting on a twin-shear element and their different effects on material failure, can comprehensively reflect the basic strength characteristics of different target materials and the influence of different strength criteria $[15,16]$, it is widely used as a more reasonable strength criterion for solving penetration problems with complex stress states [8-10]. Note that the establishment and selection of material strength criteria are crucial when studying the antipenetration performance of targets. Hence, to expand the application scope of materials and fully utilize their potential, this paper uses the unified strength theory to investigate the elastic-plastic stage and plastic stage of linear strain-hardening target materials while considering the effects of the intermediate principal stress and the free lateral boundaries of the target based on the finite cylindrical cavity expansion theory which neglects the effect of the inertia terms and the strain rate of the target material. Through this study, analytical solutions of the radial stress in the cavity wall are obtained, and a unified penetration model for linear strain-hardening target materials is built. On this basis, penetration resistance formulas and penetration depth formulas are presented for rigid projectiles penetrating into thick, finite-radius, metallic targets. Thus, the model presented in this work, which is also suitable for the penetration of rigid projectiles into semi-infinite metallic targets, has a broader scope of application and higher accuracy than previous models. Through simulations of the penetration process under different conditions with the finite element software ANSYS/LS-DYNA, the motion law of projectiles and the dynamic response of targets are studied. The analytical results in this paper are compared with the experimental and analytical results in relevant documents, which reveals that the solution in [14] is only a special case of the solution in this paper. Moreover, a series of analytical solutions based on different strength criteria are obtained to effectively predict the penetration depth ranges of all kinds of targets with equal tensile and compressive strength under different striking velocities. As the model proposed herein can be used for sharp-nosed projectiles, conical-nosed projectiles, and spherical-nosed projectiles, the effect of nose shape on the penetration depth is analysed. Moreover, the effects of the strength parameter, strength criterion, striking velocity, and target radius on the penetration depth limit are discussed and quantified. The suggestion that the condition of ignoring the effect of the free lateral boundaries of the target in target dimension design for weapons field tests is put forward through quantifying this effect on penetration performance in various working conditions. The conclusions of this study can be used as a reference of designing for metallic armor protective structures such as tanks and ships.

\section{Twin Shear Unified Strength Theory}

The twin shear unified strength theory is a new structural theory that considers the effects of the intermediate principal stress $\sigma_{2}$ and can be applied to a variety of different materials. This theory can be mathematically expressed as [17]

$$
\begin{gathered}
\sigma_{1}-\frac{\alpha}{1+b}\left(b \sigma_{2}+\sigma_{3}\right)=\sigma_{t}, \quad\left(\sigma_{2} \leq \frac{\sigma_{1}+\alpha \sigma_{3}}{1+\alpha}\right), \\
\frac{1}{1+b}\left(\sigma_{1}+b \sigma_{2}\right)-\alpha \sigma_{3}=\sigma_{t}, \quad\left(\sigma_{2} \geq \frac{\sigma_{1}+\alpha \sigma_{3}}{1+\alpha}\right),
\end{gathered}
$$

where $\sigma_{1}, \sigma_{2}$, and $\sigma_{3}$ are the maximum principal stress, intermediate principal stress, and minimum principal stress in the unit, respectively; $\alpha=\sigma_{t} / \sigma_{c}$ is the tensile strength-tocompressive strength ratio; $b=\left((1+\alpha) \tau_{s}-\sigma_{t} / \sigma_{t}-\tau_{s}\right)$ is a failure criterion parameter that reflects the influence of the intermediate principal stress, wherein $0 \leq b \leq 1$; and $\sigma_{t}, \sigma_{c}$, and $\tau_{s}$ are the tensile yield strength, compressive yield strength and shear yield strength of the material, respectively.

When taking $\alpha=1$, equations (1a) and (1b) can be simplified as

$$
\begin{gathered}
\sigma_{1}-\frac{1}{1+b}\left(b \sigma_{2}+\sigma_{3}\right)=\sigma_{s}, \quad\left(\sigma_{2} \leq \frac{\sigma_{1}+\sigma_{3}}{2}\right), \\
\frac{1}{1+b}\left(\sigma_{1}+b \sigma_{2}\right)-\sigma_{3}=\sigma_{s}, \quad\left(\sigma_{2} \geq \frac{\sigma_{1}+\sigma_{3}}{2}\right) .
\end{gathered}
$$

Since this strength theory includes infinitely many yield criteria, it is also known as the twin shear unified yield criterion [17].

\section{Finite Cylindrical Cavity Expansion Theory}

3.1. Analytical Model Based on the Unified Strength Theory. Figure 1 shows the finite cylindrical cavity expansion model [13]. The target is assumed to have a radius of $r_{t}$, a cavity radius of $r_{c}$ at time $t$, and a final cavity radius of $r_{\mathrm{cf}}$. Note that $r_{e}=c r_{c} / \dot{r}_{c}$ ( $c$ is the elastic wave velocity) is the radius of the wavefront for elastic waves, $r_{p}$ is the radius of the elastoplastic interface, and $\dot{r}_{c}$ is a constant. For incompressible materials, we can take the elastic wave velocity as $c=\infty$ and Poisson's ratio as $v=0.5$. When the cavity radius $r_{c}$ increases from 0 to $r_{\text {cf }}$, the entire expansion can be decomposed into two parts: the elastoplastic stage $\left(r_{p}<r_{t}\right)$ and the plastic stage $\left(r_{p} \equiv r_{t}\right)$. It is cracks beginning to form along the surfaces of the cylinder that can be seen as the end of the plastic stage.

Since the cylindrical cavity expansion model belongs to axisymmetrical plane strain problems, $\sigma_{z}\left(\sigma_{z}=m\left(\sigma_{r}+\right.\right.$ $\left.\sigma_{\theta}\right) / 2$ ) is the intermediate main stress, where $m$ is the 


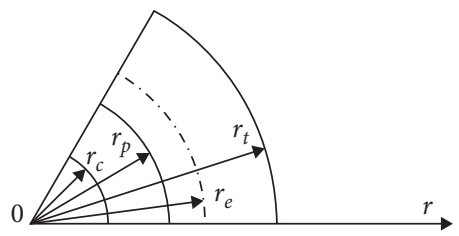

Figure 1: Finite cylindrical cavity expansion model.

intermediate main stress parameter $(0 \leq m \leq 1)$. From generalized Hooke's law, one obtains $m=2 v$ in the elastic zone and $m \approx 1$ in the plastic zone (i.e., $\left.\sigma_{z}=\left(\sigma_{r}+\sigma_{\theta}\right) / 2[18]\right)$. For incompressible materials, it is suggested that $m=1$ because the volumetric strain is 0 . If the pressure is positive, then $\sigma_{1}=\sigma_{r}, \sigma_{2}=\sigma_{z}$, and $\sigma_{3}=\sigma_{\theta}$ according to $\sigma_{1} \geq \sigma_{2} \geq \sigma_{3}$. Because $\sigma_{2} \leq\left(\sigma_{1}+\sigma_{3}\right) / 2$, the equivalent stress and strain of metal materials derived from (2a) can be written as

$$
\begin{aligned}
\sigma_{\text {eq }} & =\frac{2+b}{2(1+b)}\left(\sigma_{r}-\sigma_{\theta}\right), \\
\varepsilon_{\text {eq }} & =\frac{2(1+b) \varepsilon_{r}}{2+b},
\end{aligned}
$$

where $\sigma_{\mathrm{eq}}$ and $\varepsilon_{\mathrm{eq}}$ are the equivalent stress and strain based on the unified strength theory, respectively, $\sigma_{r}$ and $\varepsilon_{r}$ are the radial stress and strain, respectively, and $\sigma_{\theta}$ and $\varepsilon_{\theta}$ are the hoop stress and strain, respectively.

From the constitutive relations of linear hardening materials, one obtains

$$
\begin{aligned}
& \sigma=E \mathcal{\varepsilon}, \quad\left(\sigma \leq \sigma_{\text {oy }}\right), \\
& \sigma=\sigma_{\text {oy }}+E_{p}\left(\varepsilon-\frac{\sigma_{\text {oy }}}{E}\right), \quad\left(\sigma>\sigma_{\text {oy }}\right) .
\end{aligned}
$$

Combining equations (3a) and (3b) and (4a) and (4b), the relationship of effective stress-strain for linear hardening materials can be written as

$$
\begin{aligned}
& \sigma_{r}-\sigma_{\theta}=\frac{2 E}{3}\left(\varepsilon_{r}-\varepsilon_{\theta}\right), \quad\left(\sigma_{\text {eq }} \leq \sigma_{\text {oy }}\right), \\
& \sigma_{r}-\sigma_{\theta}=\frac{2(1+b) \sigma_{\text {oy }}}{2+b}\left(1-\frac{E_{p}}{E}\right)+\frac{2 E_{p} r_{c}^{2}}{3 r^{2}}, \quad\left(\sigma_{\text {eq }}>\sigma_{\text {oy }}\right) .
\end{aligned}
$$

Geometric relations in the elastic zone (small strain) and the plastic zone (large strain) are expressed as [13]

$$
\begin{aligned}
\varepsilon_{r} & =-\frac{\partial s}{\partial r}, \\
\varepsilon_{\theta} & =-\frac{s}{r}, \\
\varepsilon_{r} & =\frac{-(\partial s / \partial r)}{1-(\partial s / \partial r)}, \\
\varepsilon_{\theta} & =-\frac{s}{r-s},
\end{aligned}
$$

where $r$ and $s$ are the spatial coordinates and displacement at time $t$, respectively.

If the material density is assumed to be constant, from mass conservation, one will obtain

$$
\frac{\partial\left[(r-s)^{2}\right]}{\partial r}=2 r .
$$

Integrating the above equation and using the cavity boundary condition $r=s=r_{c}$ gives the displacement field for the particle, which can be expressed as

$$
s=r-\sqrt{r^{2}-r_{c}^{2}} .
$$

In the elastic zone, combining (6) and (9), one will obtain

$$
\varepsilon_{r}-\varepsilon_{\theta} \approx \frac{r_{c}^{2}}{r^{2}} .
$$

Substituting (10) into (5a) and (5b), the relationship of effective stress-strain for linear hardening materials is given as

$$
\begin{aligned}
& \sigma_{r}-\sigma_{\theta}=\frac{2 E r_{c}^{2}}{3 r^{2}}, \quad\left(\sigma_{\mathrm{eq}} \leq \sigma_{\mathrm{oy}}\right), \\
& \sigma_{r}-\sigma_{\theta}=\frac{2(1+b) \sigma_{\mathrm{oy}}}{2+b}\left(1-\frac{E_{p}}{E}\right)+\frac{2 E_{p} r_{c}^{2}}{3 r^{2}}, \quad\left(\sigma_{\mathrm{eq}}>\sigma_{\mathrm{oy}}\right) .
\end{aligned}
$$

The velocity field of the cylindrical cavity expansion model is expressed as [13]

$$
v=\frac{r_{c} \dot{r}_{c}}{r}
$$

The momentum conservation equation of the target in the cylindrical coordinate system can be expressed as

$$
\frac{\partial \sigma_{r}}{\partial r}+\frac{\sigma_{r}-\sigma_{\theta}}{r}=-\rho\left(\frac{\partial v}{\partial t}+v \frac{\partial v}{\partial r}\right),
$$

where $r$ is the radius of the cylinder, $t$ is the time, and $\rho$ is the density of the target material.

Equations (6), (7), and (9) and ((11a), (11b)) (13) are the fundamental equations of the finite cylindrical cavity expansion model for incompressible linear hardening materials based on unified strength theory.

\subsection{Cavity Expansion Stress Calculation}

3.2.1. Elastoplastic Stage $\left(r_{p}<r_{t}\right)$. Because of the continuity of elastoplastic boundary stresses, substituting $\left.\sigma_{\mathrm{eq}}\right|_{r=r_{p}}=\sigma_{\text {oy }}$ into (11a) and (11b), one obtains

$$
\frac{r_{c}}{r_{p}}=\sqrt{\frac{3(1+b) \sigma_{\mathrm{oy}}}{(2+b) E}} .
$$

Setting $r_{p}=r_{t}$, the radius of cavity expansion $r_{c 1}$ at the end of the first stage is expressed as 


$$
r_{c 1}=r_{t} \sqrt{\frac{3(1+b) \sigma_{\mathrm{oy}}}{(2+b) E}}
$$

In the plastic zone $\left(\sigma_{\text {eq }}>\sigma_{\text {oy }}\right)$, substituting (11a), (11b), and (12) into (13), we have

$$
\frac{\partial \sigma_{r}}{\partial r}=\frac{-2(1+b) \sigma_{\text {oy }}}{2+b}\left(1-\frac{E_{p}}{E}\right) \times \frac{1}{r}-\frac{2 E_{p} r_{c}^{2}}{3 r^{3}}-\rho\left(\frac{\dot{r}_{c}^{2}}{r}-\frac{r_{c}^{2} \dot{r}_{c}^{2}}{r^{3}}\right)
$$

Integrating (16) yields

$\sigma_{r}=\frac{-2(1+b) \sigma_{\text {oy }}}{2+b}\left(1-\frac{E_{p}}{E}\right) \ln r+\frac{E_{p} r_{c}^{2}}{3 r^{2}}-\frac{1}{2} \rho \dot{r}_{c}^{2}\left(2 \ln r+\frac{r_{c}^{2}}{r^{2}}\right)+C_{1}$,

where $C_{1}$ is a constant.

Considering the boundary condition of the cavity wall $\left.\sigma_{r}\right|_{r=r_{c}}=\sigma_{\mathrm{rc}}, C_{1}$ can be written as

$$
C_{1}=\sigma_{\mathrm{rc}}+\frac{2(1+b) \sigma_{\mathrm{oy}}}{2+b}\left(1-\frac{E_{p}}{E}\right) \ln r_{c}-\frac{E_{p}}{3}+\frac{1}{2} \rho \dot{r}_{c}^{2}\left(2 \ln r_{c}+1\right) .
$$

Then, substituting (18) into (17), the radial stress $\sigma_{r}$ is given as

$$
\begin{aligned}
\sigma_{r}= & \sigma_{\mathrm{rc}}+\frac{(1+b) \sigma_{\mathrm{oy}}}{2+b}\left(1-\frac{E_{p}}{E}\right) \ln \frac{r_{c}^{2}}{r^{2}}-\frac{E_{p}}{3}+\frac{E_{p} r_{c}^{2}}{3 r^{2}} \\
& +\frac{1}{2} \rho \dot{r}_{c}^{2}\left(1+\ln \frac{r_{c}^{2}}{r^{2}}-\frac{r_{c}^{2}}{r^{2}}\right) .
\end{aligned}
$$

Considering the elastoplastic boundary condition $\sigma_{r} \mid r=r_{p}=\sigma_{\mathrm{rp}}^{-}$, the radial stress in the cavity wall in the elastoplastic stage $\sigma_{\text {rc }}$ derived from (19) can be written as

$$
\begin{aligned}
\sigma_{\mathrm{rc}}= & \sigma_{\mathrm{rp}}^{-}+\frac{E_{p}}{3}\left(1-\frac{r_{c}^{2}}{r_{p}^{2}}\right)-\frac{\sigma_{\mathrm{oy}}(1+b)}{2+b} \frac{\left(E-E_{p}\right)}{E} \ln \frac{r_{c}^{2}}{r_{p}^{2}} \\
& -\frac{1}{2} \rho \dot{r}_{c}^{2}\left(1+\ln \frac{r_{c}^{2}}{r_{p}^{2}}-\frac{r_{c}^{2}}{r_{p}^{2}}\right),
\end{aligned}
$$

where $\sigma_{\mathrm{rp}}^{-}$is the radial stress in the plastic zone side of the elastoplastic boundary.

In the elastic zone $\left(\sigma_{\mathrm{eq}} \leq \sigma_{\mathrm{oy}}\right)$, substituting (11a), (11b), and (12) into (13), we have

$$
\frac{\partial \sigma_{r}}{\partial r}=-\frac{2 E r_{c}^{2}}{3 r^{3}}-\rho \dot{r}_{c}^{2}\left(\frac{1}{r}-\frac{r_{c}^{2}}{r^{3}}\right)
$$

Integrating (21), one obtains

$$
\sigma_{r}=\frac{E r_{c}^{2}}{3 r^{2}}-\frac{1}{2} \rho \dot{r}_{c}^{2}\left(2 \ln r+\frac{r_{c}^{2}}{r^{2}}\right)+C_{2}
$$

where $C_{2}$ is a constant.

Considering the free lateral boundaries of the cylinder $\left.\sigma_{r}\right|_{r=r_{t}}=0$ and (22), one can obtain

$$
C_{2}=-\frac{E r_{c}^{2}}{3 r_{t}^{2}}+\frac{1}{2} \rho \dot{r}_{c}^{2}\left(2 \ln r_{t}+\frac{r_{c}^{2}}{r_{t}^{2}}\right)
$$

Substituting (23) into (22), we obtain the radial stress in the elastic zone $\sigma_{r}$ as

$$
\sigma_{r}=\frac{E}{3}\left(\frac{r_{c}^{2}}{r^{2}}-\frac{r_{c}^{2}}{r_{t}^{2}}\right)+\frac{1}{2} \rho \dot{r}_{c}^{2}\left(\ln \frac{r_{t}^{2}}{r^{2}}+\frac{r_{c}^{2}}{r_{t}^{2}}-\frac{r_{c}^{2}}{r^{2}}\right)
$$

Considering the elastoplastic boundary condition $\left.\sigma_{r}\right|_{r=r_{p}}=\sigma_{\mathrm{rp}}^{+}$and (19), one obtains

$$
\sigma_{\mathrm{rp}}^{+}=\frac{E}{3}\left(\frac{r_{c}^{2}}{r_{p}^{2}}-\frac{r_{c}^{2}}{r_{t}^{2}}\right)+\frac{1}{2} \rho \dot{r}_{c}^{2}\left(\ln \frac{r_{t}^{2}}{r_{p}^{2}}+\frac{r_{c}^{2}}{r_{t}^{2}}-\frac{r_{c}^{2}}{r_{p}^{2}}\right),
$$

where $\sigma_{\mathrm{rp}}^{+}$is the radial stress in the elastic zone side of the elastoplastic boundary.

Because of the continuity conditions $\sigma_{\mathrm{rp}}^{-}=\sigma_{\mathrm{rp}}^{+}$, substituting (25) into (20), one obtains

$$
\begin{aligned}
\sigma_{\mathrm{rc}}= & \frac{\left(E-E_{p}\right) r_{c}^{2}}{3 r_{p}^{2}}+\frac{E_{p}}{3}-\frac{E r_{c}^{2}}{3 r_{t}^{2}}-\frac{\sigma_{\text {oy }}(1+b)\left(E-E_{p}\right)}{(2+b) E} \\
& \times \ln \frac{r_{c}^{2}}{r_{p}^{2}}+\frac{1}{2} \rho \dot{r}_{c}^{2}\left(\ln \frac{r_{t}^{2}}{r_{c}^{2}}+\frac{r_{c}^{2}}{r_{t}^{2}}-1\right) .
\end{aligned}
$$

Substituting (14) into (26), the radial stress in the cavity wall in the elastoplastic stage $\sigma_{\mathrm{rcl}}$ can be written as

$$
\begin{aligned}
\sigma_{\mathrm{rcl}}= & \frac{\sigma_{\mathrm{oy}}(1+b)}{2+b}\left[1-\frac{E-E_{p}}{E} \ln \frac{3(1+b) \sigma_{\mathrm{oy}}}{(2+b) E}\right] \\
& +\frac{E_{p}}{3} \times\left[1-\frac{3(1+b) \sigma_{\mathrm{oy}}}{(2+b) E}\right] \\
& -\frac{E r_{c}^{2}}{3 r_{t}^{2}}+\frac{1}{2} \rho \dot{r}_{c}^{2}\left(\ln \frac{r_{t}^{2}}{r_{c}^{2}}+\frac{r_{c}^{2}}{r_{t}^{2}}-1\right), \quad\left(r_{c} \leq r_{c 1}\right) .
\end{aligned}
$$

3.2.2. Plastic Stage $\left(r_{p} \equiv r_{t}\right)$. Substituting the boundary condition $\left.\sigma_{r}\right|_{r=r_{t}}=0$ into (19), the radial stress in the cavity wall in the plastic stage $\sigma_{\mathrm{rc} 2}$ can be given as 


$$
\begin{aligned}
\sigma_{\mathrm{rc} 2}= & \frac{E_{p}}{3}-\frac{E_{p} r_{c}^{2}}{3 r_{t}^{2}}+\frac{\sigma_{\mathrm{oy}}(1+b)\left(E-E_{p}\right)}{(2+b) E} \ln \frac{r_{t}^{2}}{r_{c}^{2}} \\
& +\frac{1}{2} \rho \dot{r}_{c}^{2}\left(\ln \frac{r_{t}^{2}}{r_{c}^{2}}+\frac{r_{c}^{2}}{r_{t}^{2}}-1\right), \quad\left(r_{c 1}<r_{c} \leq r_{c 2}\right),
\end{aligned}
$$

where $r_{c 2}$ is the cavity radius at the end of the second stage.

According to $\sigma_{r}=0$ when the boundary condition $r=r_{t}$, we obtain $\sigma_{2} \leq\left(\sigma_{1}+\alpha \sigma_{3} / 1+\alpha\right)$ from (1a) and (1b), and the equivalent stress based on unified strength theory $\sigma_{\text {eq }}$ can be computed as

$$
\sigma_{\text {eq }}=\frac{2+2 b-\alpha b}{2(1+b)} \sigma_{r}-\frac{2 \alpha+\alpha b}{2(1+b)} \sigma_{\theta}=-\frac{2 \alpha+\alpha b}{2(1+b)} \sigma_{\theta} .
$$

Based on the hypothesis of plastic work $[19,20]$ and the stress-strain state of the finite cylindrical cavity expansion problem, one obtains

$$
\sigma_{\mathrm{eq}} \varepsilon_{\mathrm{eq}}=\sigma \mathcal{\varepsilon}=\sigma_{\theta} \varepsilon_{\theta},
$$

where $\varepsilon_{\mathrm{eq}}$ is the equivalent strain based on unified strength theory. Combining (29) and (30), this equivalent strain can be given as

$$
\varepsilon_{\mathrm{eq}}=-\frac{2(1+b) \varepsilon_{\theta}}{(2 \alpha+\alpha b)} .
$$

Substituting (9) into (7), the strain components can be expressed as

$$
\varepsilon_{r}=-\varepsilon_{\theta} \approx \frac{1}{2}\left(\frac{r_{c}}{r}\right)^{2} .
$$

Combining (31) and (32) and considering the fracture criterion $\left.\varepsilon_{\text {eq }}\right|_{r=r_{t}}=\varepsilon_{f}$ in [21], the cavity radius $r_{c 2}$ at the end of the second stage when $\alpha=1$ can be expressed as

$$
r_{c 2}=r_{t} \sqrt{\frac{(2+b) \varepsilon_{f}}{1+b}}
$$

where $\varepsilon_{f}$ is the fracture strain of the target material under uniaxial tension.

\subsection{Penetration of Rigid Projectiles into a Thick Cylindrical Metallic Target}

3.3.1. Penetration Model Analysis for Rigid Projectiles. Suppose that the generatrix equation for the rotating warhead of a rigid sharp-nosed projectile is expressed as $y=y(x)$, where $a$ and $l$ are the radius and nose length of the projectile, respectively, as shown in Figure 2. Accordingly, a

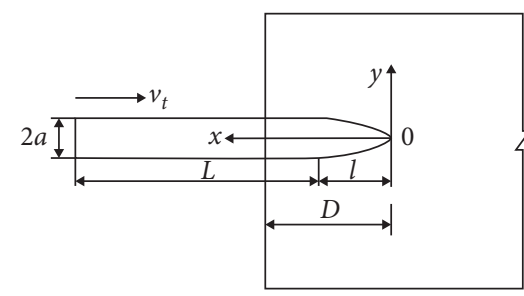

Figure 2: Dimensions of penetration model.

simulation of the penetration process of the projectile can be regarded as a series of finite cylindrical cavity expansion models with a cavity radius of $r_{c}=y(x)$ and a target radius of $r_{t}$ [14]. Assuming the initial velocity of the projectile is $v_{0}$ and the initial penetration depth of the target is $D_{0}$, the velocity of the projectile is $v_{t}$ and the penetration depth of the target is $D_{t}$ at time $t$; the radius of the target cavity $r_{c}$ and the velocity of cavity expansion $v_{t}$ at time $t$ can be expressed as [14]

$$
\begin{gathered}
r_{c}=y(x), \\
\dot{r}_{c}=y^{\prime}(x) v_{t} .
\end{gathered}
$$

3.3.2. Penetration Resistance Calculation. Ignoring the variation in resistance when $D<l$, when $D \geq l$, the penetration resistance can be expressed as [14]

$$
\begin{gathered}
F_{x}=\int_{0}^{l} \sigma_{r}(x) \times \frac{2 \pi y(x) \mathrm{d} x}{\cos \varphi} \times \sin \varphi \\
=2 \pi \int_{0}^{l} y(x) y^{\prime}(x) \sigma_{r}(x) \mathrm{d} x,
\end{gathered}
$$

where $\varphi$ is the angle between the tangent of a point on the projectile arc and the projectile axis and $\sigma_{r}(x)$ is the radial stress in the surface of the projectile calculated by the analytical model in this paper.

Rigid projectiles can be divided into three types according to their nose shape: conical-nosed projectiles, sharp-nosed projectiles, and spherical-nosed projectiles, as shown in Figure 3. For conical-nosed projectiles, sharpnosed projectiles with $\mathrm{CRH}=3(\mathrm{CRH}$ is the ratio between the arc radius of the projectile nose and the diameter of the projectile), and spherical-nosed projectiles, the cavity radius can be expressed as $y(x)=(a / l) x, \quad y(x)=$ $\sqrt{36 a^{2}-(x-l)^{2}}-5 a, \quad$ and $\quad y(x)=\sqrt{a^{2}-(x-l)^{2}}$, respectively.

Substituting (27) and (28) into (35), one obtains 


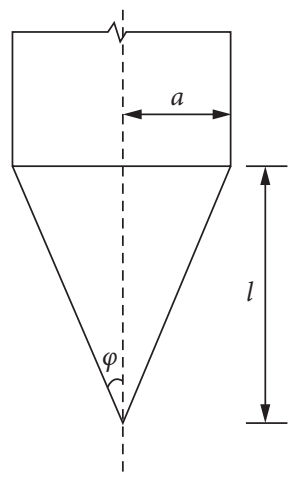

(a)

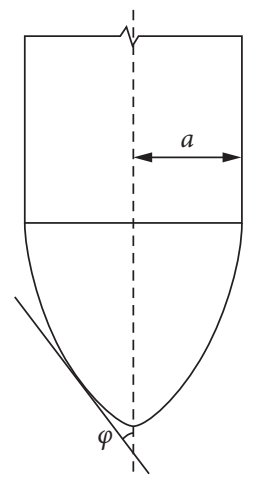

(b)

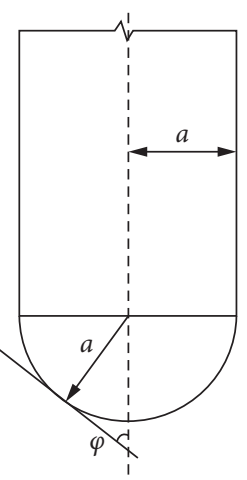

(c)

Figure 3: Rigid projectiles with different nose shapes, (a) conical-nosed, (b) sharp-nosed, and (c) spherical-nosed.

$$
\begin{aligned}
F_{x}= & 2 \pi \int_{0}^{l} y(x) y^{\prime}(x) \times\left\{\frac { \sigma _ { \text { oy } } ( 1 + b ) } { 2 + b } \left[1-\frac{E-E_{p}}{E} \ln \frac{3(1+b) \sigma_{\text {oy }}}{(2+b) E}\right.\right. \\
& +\frac{E_{p}}{3}\left[1-\frac{3(1+b) \sigma_{\text {oy }}}{(2+b) E}\right]-\frac{E r_{c}^{2}}{3 r_{t}^{2}} \\
& \left.+\frac{1}{2} \rho \dot{r}_{c}^{2}\left(\ln \frac{r_{t}^{2}}{r_{c}^{2}}+\frac{r_{c}^{2}}{r_{t}^{2}}-1\right)\right\} \mathrm{d} x, \quad\left(r_{c} \leq r_{c 1}\right),
\end{aligned}
$$$$
F_{x}=2 \pi \int_{0}^{l} y(x) y^{\prime}(x) \times\left\{\frac{E_{p}}{3}-\frac{E_{p} r_{c}^{2}}{3 r_{t}^{2}}+\frac{\sigma_{\text {oy }}(1+b)}{(2+b)} \times \frac{\left(E-E_{p}\right)}{E}\right.
$$$$
\left.\times \ln \frac{r_{t}^{2}}{r_{c}^{2}}+\frac{1}{2} \rho \dot{r}_{c}^{2}\left(\ln \frac{r_{t}^{2}}{r_{c}^{2}}+\frac{r_{c}^{2}}{r_{t}^{2}}-1\right)\right\} \mathrm{d} x, \quad\left(r_{c 1}<r_{c} \leq r_{c 2}\right),
$$

where $\quad r_{c 1}=r_{t} \sqrt{\left(3(1+b) \sigma_{\mathrm{oy}} /(2+b) E\right)} \quad$ and $\quad r_{c 2}=r_{t}$ $\sqrt{\left((2+b) \varepsilon_{f} / 1+b\right)}$.

Supposing $y\left(x_{1}\right)=r_{c 1}$ at the end of the elastoplastic stage, $a \leq r_{c 1}$; hence, the penetration resistance can be calculated by (36a) because the finite cylindrical cavity expansion model only undergoes the elastoplastic stage when $x_{1} \geq l$. In contrast, when $a>r_{c 1}$, the penetration resistance can be calculated in sections by (36a) and (36b) because the finite cylindrical cavity expansion model undergoes the elastoplastic stage and the plastic stage fully when $x_{1}<l$.

Combining (34a) and (34b) and (36a) and (36b), formulas with the same form as those in [14] can be given as

$$
\begin{aligned}
& F_{x}=F_{s}+F_{d}=\pi a^{2} \sigma_{s}+\pi a^{2} N_{d} \rho v_{t}^{2}, \\
& \sigma_{s}=A_{1} N_{1}+A_{2} N_{2}+B_{1} M_{1}+B_{2} M_{2}+A_{2}^{*} N_{2}^{*},
\end{aligned}
$$

where $F_{s}$ and $F_{d}$ are the static strength resistance and flow resistance, respectively; $A_{1}, B_{1}, N_{1}$, and $M_{1}$ are the static strength coefficients related to the performance of the projectile and target materials in the elastoplastic stage; $A_{2}, B_{2}, N_{2}, M_{2}, A_{2}^{*}$, and $N_{2}^{*}$ are the static strength coefficients related to the performance of the projectile and target materials in the plastic stage; and $N_{d}$ is the coefficient of the flow resistance. Note that projectiles with different nose shapes have different values of $N_{d}$.

The coefficients in (37) and (38) can be calculated as

$$
\begin{aligned}
A_{1}= & \frac{\sigma_{\text {oy }}(1+b)}{2+b}\left[1-\frac{E-E_{p}}{E} \ln \frac{3(1+b) \sigma_{\text {oy }}}{(2+b) E}\right] \\
& +\frac{E_{p}}{3}\left[1-\frac{3(1+b) \sigma_{\text {oy }}}{(2+b) E}\right]
\end{aligned}
$$

$$
\begin{aligned}
& A_{2}=\frac{E_{p}}{3} ; \\
& B_{1}=-\frac{E}{3 r_{t}^{2}} ; \\
& B_{2}=-\frac{E_{p}}{3 r_{t}^{2}} ;
\end{aligned}
$$

$A_{2}^{*}=\frac{2 \sigma_{\text {oy }}(1+b)\left(E-E_{p}\right)}{(2+b) E}$

$N_{d}=\frac{1}{a^{2}} \int_{0}^{l} y(x)\left[y^{\prime}(x)\right]^{3} \times\left(\ln \frac{r_{t}^{2}}{[y(x)]^{2}}+\frac{[y(x)]^{2}}{r_{t}^{2}}-1\right) \mathrm{d} x ;$

$N_{1}= \begin{cases}1, & \left(x_{1} \geq l\right), \\ \frac{r_{c 1}^{2}}{a^{2}}, & \left(x_{1}<l\right),\end{cases}$

$N_{2}= \begin{cases}0, & \left(x_{1} \geq l\right), \\ \frac{a^{2}-r_{c 1}^{2}}{a^{2}}, & \left(x_{1}<l\right),\end{cases}$

$M_{1}= \begin{cases}\frac{a^{2}}{2}, & \left(x_{1} \geq l\right), \\ \frac{r_{c 1}^{4}}{2 a^{2}}, & \left(x_{1}<l\right),\end{cases}$

$M_{2}= \begin{cases}0, & \left(x_{1} \geq l\right), \\ \frac{a^{4}-r_{c 1}^{4}}{2 a^{2}}, & \left(x_{1}<l\right),\end{cases}$ 


$$
N_{2}^{*}= \begin{cases}0, & \left(x_{1} \geq l\right), \\ \ln \frac{r_{t}}{a}-\frac{r_{c 1}^{2}}{a^{2}} \ln \frac{r_{t}}{r_{c 1}}-\frac{r_{c 1}^{2}}{2 a^{2}}+\frac{1}{2}, & \left(x_{1}<l\right),\end{cases}
$$

where $N_{d}$ can be determined with the method of digital integration using the Simpson formula.

3.4. Penetration Depth Calculation. Supposing the projectile mass is $m$, according to [14], the penetration depth $D$ of a projectile penetrating into a finite-radius metallic target at time $t$ is derived from Newton's second law and the initial conditions, which can be expressed as

$$
D=\frac{m}{2 \pi a^{2} \rho N_{d}} \times \ln \left(\frac{v_{0}^{2}+\left(\sigma_{s} / \rho N_{d}\right)}{v_{t}^{2}+\left(\sigma_{s} / \rho N_{d}\right)}\right) .
$$

Considering the penetration terminating condition $\left.D\right|_{v_{t}=0}=D_{\max }$, the above formula can be written as

$$
D_{\max }=\frac{m}{2 \pi a^{2} \rho N_{d}} \times \ln \left(\frac{\rho N_{d}}{\sigma_{s}} v_{0}^{2}+1\right) .
$$

3.5. Experimental Verification. Substituting the corresponding test data in [12] into the formulas in this paper, for the target, $r_{t}=127 \mathrm{~mm}$ (i.e., the target radius of 6061-T6511 aluminium alloy in the test), $E=68.9 \mathrm{GPa}$ (elastic modulus), $E_{p}=46 \mathrm{MPa}$ (tangent modulus), $\sigma_{\text {oy }}=365 \mathrm{MPa}$ (initial yield stress), and $\rho=2710 \mathrm{~kg} / \mathrm{m}^{3}$ (density). Comparisons between the analytical results and the test results in the document are summarized in Table 1.

Figure 4 compares the ultimate penetration depth results calculated with the formulas in this paper when $b=0.4$, the test results in [12] and the results calculated with the Forrestal formula [14] for different values of the projectile striking velocity $v_{0}$. The mean relative deviation between the results from the theoretical formula derived in this paper and the test results is 0.0094 , and the corresponding mean square deviation is 0.0427 . In contrast, the mean relative deviation between the Forrestal formula results [14] and the test results is 0.1469 , and the corresponding mean square deviation is 0.0669 . Accordingly, the theoretical values in this paper have a $13.75 \%$ smaller deviation from the test results than the Forrestal formula results. Hence, the results calculated with the formulas in this paper match the test results well and have higher precision than the results calculated with the Forrestal formula.

\subsection{Parameter Discussion}

3.6.1. Strength Parameter $b$ and Striking Velocity $v_{0}$. Since different materials have different values of $b$, we can obtain a series of different penetration depth and penetration resistance solutions for which the unified strength theory can be applied to various materials. Figure 5 shows the relation curves between the ultimate penetration depth $D_{\max }$ and the striking velocity $v_{0}$ for different values of the strength parameter $b$, which reveals that the strength parameter $b$ has obvious influences on the ultimate penetration depth: the higher the value of $b$ is, the more obvious the intermediate principal stress effect is and the smaller the value of $D_{\max }$ is. Hence, the value of $b$ can objectively indicate the strength potential of the material and ensure the penetration resistance of the material is fully utilized due to the consideration of the intermediate principal stress effect. Thus, this parameter should not be neglected in penetration analyses and associated calculations; otherwise, the accuracy of precision guided weapon designs will be affected.

Because the unified strength theory parameter $b$ represents the selection of different strength criteria, when $b$ takes different values, the unified strength theory can degenerate into different strength criteria (e.g., it can degenerate into the Tresca yield criterion when $b=0$, the von Mises yield criterion when $b=0.366$, and the Twin shear stress yield criterion when $b=1$ ), for which there are large differences among the computed results. Taking $v_{0}=569 \mathrm{~m} / \mathrm{s}$ as an example, the penetration depth calculated based on the Tresca yield criterion $(b=0)$ is $22.45 \%$ higher than that calculated based on the Twin shear yield criterion $(b=1)$. This finding shows that the selection of the strength criteria plays an important role in predicting the penetration effects. Thus, a suitable strength criterion should be selected for practical engineering designs.

For the penetration of thick, finite-radius, metallic targets under special conditions, unlike other calculation methods with a unique solution, the calculation method in this paper can obtain a series of analytical solutions. The results in [14] (von Mises yield criterion results), which are only applicable for materials with $\tau_{s}=0.577 \sigma_{s}$, are only a special case of the results in this paper when $b=0.366$. Moreover, nearly all of the experimental values fall within the range of the abovementioned series of analytical solutions. Therefore, under special conditions, the lower limit and upper limit of the penetration depth according to the calculation method in this paper can be used to effectively predict the range of penetration depth. For instance, the penetration depth ranges of all kinds of targets with equal tensile strength and compressive strength under different striking velocities are determined, and the results are summarized in Table 2 .

In addition, from Figure 5, we can also see that when $v_{0} \leq 1147 \mathrm{~m} / \mathrm{s}$ (meeting the rigid penetration conditions in this paper), the higher the striking velocity of the projectile is, the greater the penetration depth is.

Figure 6 shows the penetration depth $D$ versus the projectile penetration velocity $v_{t}$ for different projectile striking velocities $v_{0}$, from which we can see that as the penetration process elapses, the projectile velocity continuously decreases and the penetration depth continuously increases; however, the rate of increase in the penetration depth decreases over time.

3.6.2. Ratio of the Target Radius to the Projectile Radius $r_{t} / a$. Hereafter, for comparison with the ballistic tests results, the variation in the penetration depth is studied under different projectile striking velocities $v_{0}$ and target radii (i.e., different 
TABLE 1: Comparisons of the analytical results of penetration depth.

\begin{tabular}{|c|c|c|c|c|c|c|c|}
\hline$m(\mathrm{~g})$ & $a(\mathrm{~mm})$ & $l(\mathrm{~mm})$ & $L(\mathrm{~mm})$ & $H(\mathrm{~mm})$ & $v_{0}\left(\mathrm{~ms}^{-1}\right)$ & $D_{\text {shi }}(\mathrm{mm})$ & $D_{\max }(\mathrm{mm})$ \\
\hline 20.38 & 3.555 & 11.8 & 59.3 & 127 & 569 & 58 & 58.33 \\
\hline 20.43 & 3.555 & 11.8 & 59.3 & 141 & 570 & 55 & 58.64 \\
\hline 20.41 & 3.555 & 11.8 & 59.3 & 140 & 679 & 72 & 78.60 \\
\hline 20.42 & 3.555 & 11.8 & 59.3 & 167 & 821 & 102 & 106.18 \\
\hline 20.42 & 3.555 & 11.8 & 59.3 & 229 & 966 & 140 & 135.06 \\
\hline 20.41 & 3.555 & 11.8 & 59.3 & 248 & 1147 & 190 & 170.88 \\
\hline 20.91 & 3.555 & 11.8 & 59.3 & 154 & 794 & 103 & 103.29 \\
\hline 20.89 & 3.555 & 11.8 & 59.3 & 230 & 1076 & 160 & 160.59 \\
\hline
\end{tabular}

Notes. $m$ is the mass of the projectile, $a, l$, and $L$ are the geometric dimensions of the projectile as shown in Figure $2, H$ is the thickness of the target, $v_{0}$ is the striking velocity of projectile, $D_{\text {shi }}$ is the ultimate penetration depth of the test in [12], and $D_{\max }$ is the ultimate penetration depth from the theoretical formula derived in this paper.

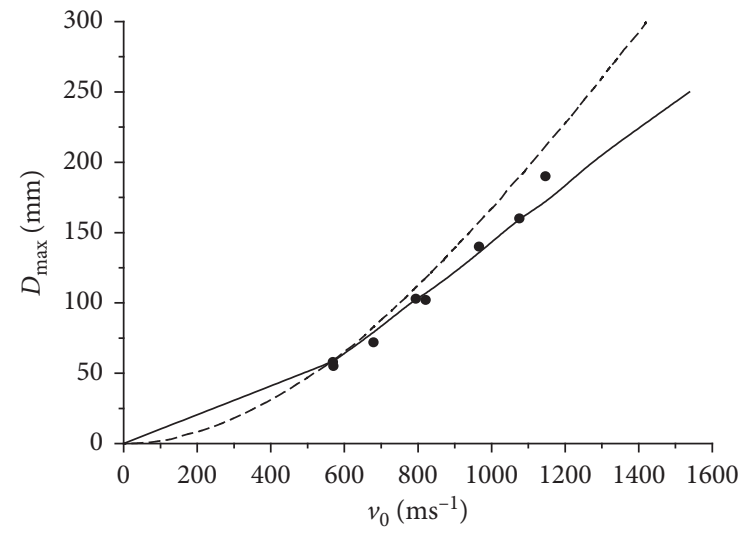

- $\quad$ Test results in [12]

- The Forrestal formula results in [14]

$\rightarrow$ Calculating results of theoretical formulas in this paper

Figure 4: Comparison of penetration depth.

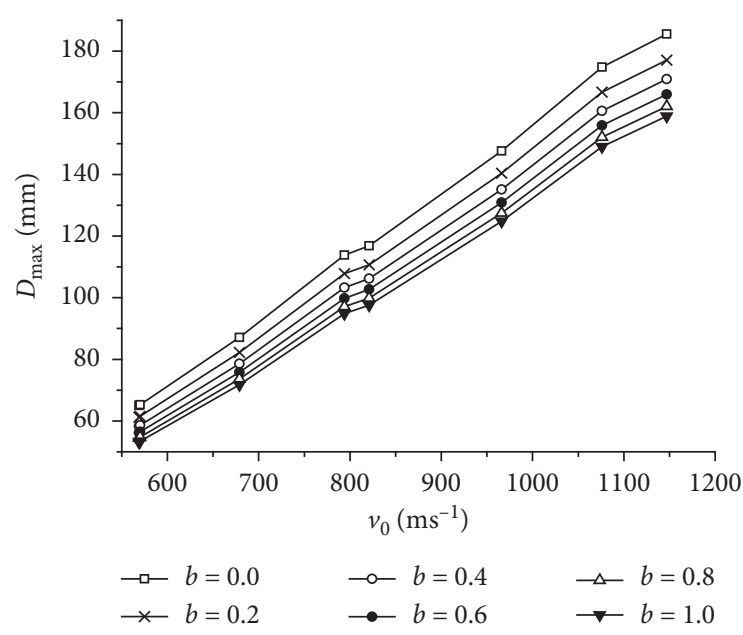

FIGURE 5: Comparison of penetration depth for different values of $b$.

$\left.r_{t} / a\right)$ while keeping the other conditions the same as those in the tests, as shown in Figure 7. During the calculation, when $r_{t} / a \leq 10, a \leq r_{c 1}$, and the finite cylindrical cavity expansion model undergoes the elastoplastic stage and the plastic stage fully; hence, the penetration resistance can be calculated in sections by (36a) and (36b). In contrast, when $r_{t} / a>10$ and $a>r_{c 1}$, and the finite cylindrical cavity expansion model only undergoes the elastoplastic stage; hence, the penetration resistance can be calculated by (36a). Figure 7 shows that as the target radius-to-projectile radius ratio increases, the penetration depth-to-projectile radius ratio decreases, but the rate of decrease slows when $r_{t} / a \geq 16$. Compared to the penetration depth when $r_{t} / a=16$, the penetration depth when $r_{t} / a=70$ is reduced by only $3.15 \%$, whereas when $r_{t} / a=4$, the penetration depth is increased by $40.76 \%$. These findings indicate that the influence of the target boundary size cannot be neglected (i.e., the calculations cannot still assume that the target has an unlimited-size) when $r_{t} / a<16$, which is in good agreement with the experimental results in [12].

3.6.3. Projectile Nose Shape. Figure 8 shows a comparison of the ultimate penetration depth $D_{\max }$ of a sharp-nosed projectile, a conical-nosed projectile, and a spherical-nosed projectile (the diameter of each column body is the same) under different projectile striking velocities $v_{0}$. It is quite evident that the projectile nose shape has an obvious influence on the antipenetration performance of thick, finiteradius, metallic targets. The penetration depth of the conical-nosed projectile is greater than that of the sharpnosed projectile and is substantially greater than that of the spherical-nosed projectile. When the striking velocity is less than approximately $570 \mathrm{~m} / \mathrm{s}$, the penetration depth of the sharp-nosed projectile and that of the conical-nosed projectile are approximately equal. Although the column body diameters and lengths are equal, the spherical-nosed projectile, which has a flat nose and a relatively large surface area, experiences a greater amount of resistance during the penetration process than the other two projectile types. This indicates that the penetration capacities of the sharp-nosed projectile and conical-nosed projectile are obviously much stronger than that of the spherical-nosed projectile under the same conditions, and the sharp-nosed projectile has the strongest penetration capacity. There is little difference between the penetration capacity of the sharp-nosed projectile and that of the conical-nosed projectile when the striking velocity is less than approximately $570 \mathrm{~m} / \mathrm{s}$. 
TABLE 2: Penetration depth ranges.

\begin{tabular}{lccccccc}
\hline \multirow{2}{*}{$v_{0}\left(\mathrm{~ms}^{-1}\right)$} & \multicolumn{9}{c}{$D_{\max }(\mathrm{mm})$} \\
& $b=0$ & $b=0.2$ & $b=0.4$ & $b=0.6$ & $b=0.8$ & $b=1.0$ & Penetration depth range (mm) \\
\hline 569 & 64.98 & 61.15 & 58.33 & 56.17 & 54.46 & 53.07 & $53.1 \sim 65.0$ \\
570 & 65.32 & 61.47 & 58.64 & 56.47 & 54.75 & 53.35 & $53.4 \sim 65.3$ \\
679 & 87.08 & 82.20 & 78.60 & 75.82 & 73.62 & 71.83 & $71.8 \sim 87.1$ \\
794 & 113.80 & 107.77 & 103.29 & 99.83 & 97.08 & 94.83 & $94.8 \sim 113.8$ \\
821 & 116.84 & 110.72 & 106.18 & 102.67 & 99.87 & 97.59 & $97.6 \sim 116.8$ \\
966 & 147.64 & 140.44 & 135.06 & 130.88 & 127.54 & 124.81 & $124.8 \sim 124.8$ \\
1076 & 174.77 & 166.67 & 160.60 & 155.86 & 152.08 & 148.97 & $149.0 \sim 174.8$ \\
1147 & 185.47 & 177.14 & 170.88 & 166.01 & 162.10 & 158.89 & $158.9 \sim 185.5$ \\
\hline
\end{tabular}

Note. $v_{0}$ is the striking velocity of projectile, $D_{\max }$ is the ultimate penetration depth from the theoretical formula derived in this paper, and $b$ is the strength parameter.

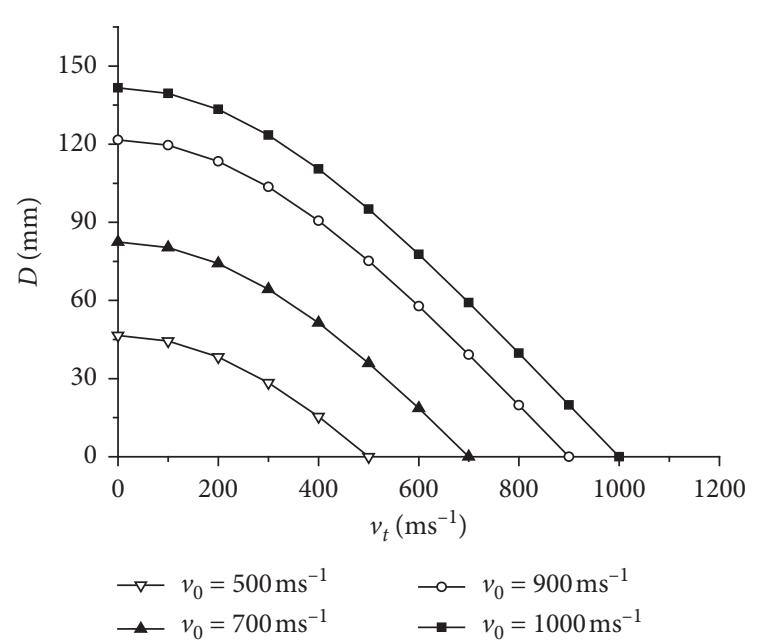

FIGURE 6: Relationship between penetration depth and penetration velocity.

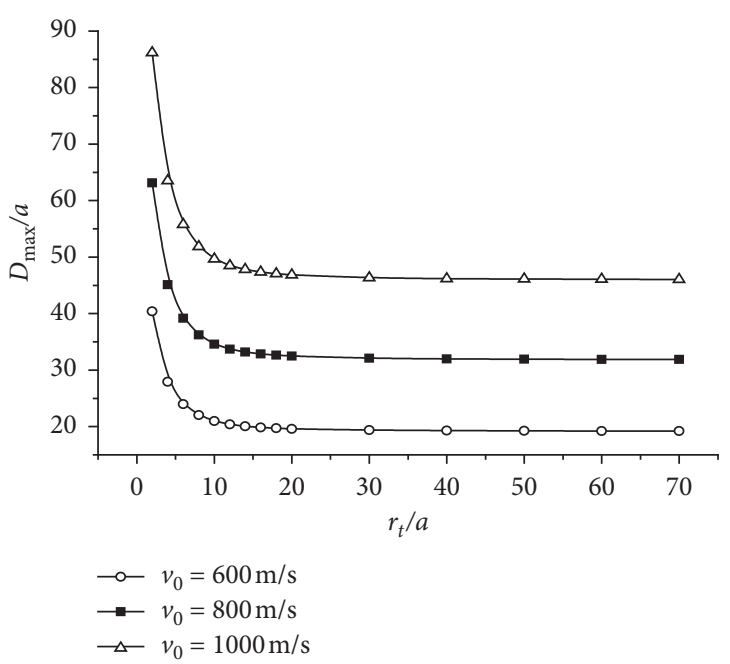

FIGURE 7: Relationship between the penetration depth and the target radius.

Figure 8 also shows that all penetration depths increase with increasing projectile striking velocity. However, the penetration depths of the sharp-nosed projectile and conical-nosed projectile are more substantially affected by the striking velocity than that of the spherical-nosed projectile.

\section{Numerical Simulation}

To further analyse the effect of the free lateral boundaries of the target on penetration into thick, finite-radius, metallic targets and investigate the projectile/target morphologies, the penetration process, and the instantaneous response, the software LS-DYNA is applied to carry out three-dimensional numerical calculations, which can greatly reduce experimental expenses and allow data and phenomena to be observed that cannot be obtained through other methods [22].

4.1. Model Verification and Results Analysis. The target centreline is the initial contact point between the projectile and the target, leaving a gap between them to eliminate the initial permeability. Considering the amount and efficiency of calculation, quarter-models of the entities are established by using the Lagrange method for the numerical simulations based on the ballistic test parameters in [12], as shown in Figure 9 (take member 1 for example). SOLID164 elements are adopted to mesh the projectile and the target; these elements have eight nodes, wherein every node has nine degrees of freedom. For the projectile, there are 11657 nodes and 9728 elements in total. For the target, there are 96128 nodes and 88900 elements in total, which divide the target evenly along the thickness in 127 layers and divide the target unevenly along the cross section in 40 regions. The meshing is fine near the centre of the target, whereas it is coarse near the boundaries: the elements near the boundaries are 2.175 times the size of those in the centre. In addition, a symmetric boundary constraint is applied on the symmetric plane.

Since the experimental results showed that the projectile did not sustain any obvious deformation or mass loss when the striking velocity was less than $1240 \mathrm{~m} / \mathrm{s}$, it can be considered to be rigid and defined by the rigid material model *MAT_RIGID, whereas the Johnson-Cook model is adopted to define the target. Moreover, using *EOS_GRUNEISEN and equivalent plastic strain as the state equation and failure criterion, respectively, when the equivalent plastic strain of the element reaches the critical 


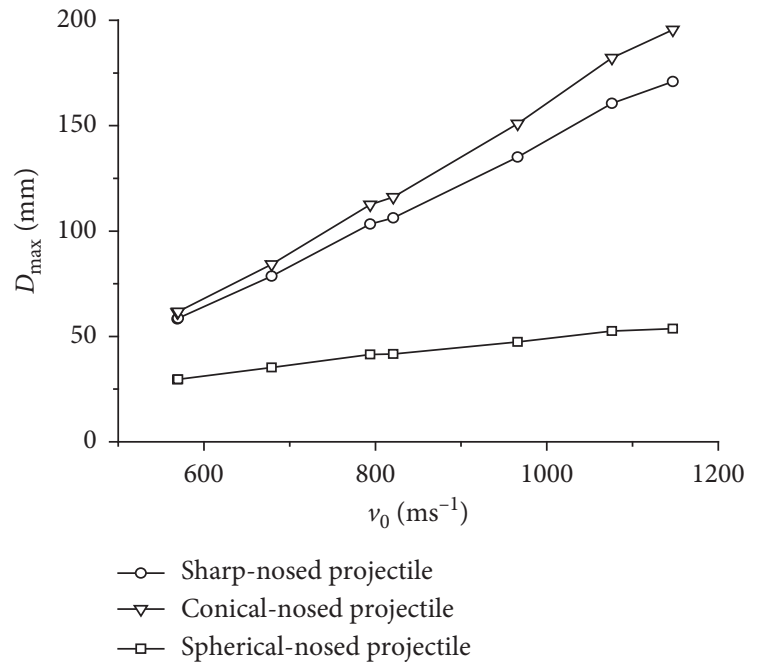

Figure 8: Comparison of penetration depth with different projectile nose shapes.

strain, this element is considered to be damaged and can be deleted. The parameters of the material model are shown in Table 3.

The velocity of the projectile showed an extremely small negative value caused by its few rebound at the end of the penetration. In order to increase efficiency, we set the total time to $0.5 \mathrm{~ms}$ because the maximum penetration depth of models all appeared, the velocity of the projectile was 0 and the projectile appeared rebound at this moment, and this velocity (very small negative value) was far less than $2 \%$ of the initial velocity.

Figure 10 shows a comparison between the numerical simulation results (the ultimate penetration depth $D_{\max }$ ) in this paper and the test results in the document for different projectile striking velocities $v_{0}$. From this comparison, we can see that the mean relative deviation between the calculated results and test results is 0.0265 , and the corresponding mean square deviation is 0.0537 . Accordingly, this error is less than that for the Forrestal formula calculations. This greater agreement with the test results indicates that the numerical model and the material parameters adopted in this paper are more reasonable and effective.

The results of the numerical simulations based on the material parameters of member 1 are plotted in Figures 11 and 12. From Figure 12(b), we can intuitively see that the stress wave that spreads from the contacting point between the rod and target will arrive at the lateral boundaries of the target in a short time (if we set the total time to $0.5 \mathrm{~ms}$, this time will be $t=34 \mu \mathrm{s}$ for a target with $r_{t} / a=10$ ). However, then, the stress wave will reflect and spread back and forth constantly between the projectile and the target. In the process of the projectile contacting the target, entering the target, and finally stopping, the highest stress occurs at the point of contact, which is the reason that the material between the projectile and target is easily destroyed.

Figure 13 shows the time-history curves of the penetration depth $D$ and projectile velocity $v_{t}$. It is quite evident that the projectile velocity decreases continuously over time, during which the penetration depth increases correspondingly. This increase in penetration depth is faster in the early stage before stabilizing.

Hereafter, to get the approximate influence curve of the penetration caused by the target size while taking computer's computational efficiency into consideration, the size of the target is changed $\left(r_{t} / a=2.5,5,10,15,20,35.7\right.$, and 60$)$ while keeping the other parameters consistent with those of member 2 to investigate the influence of different target radius-to-projectile radius ratios on the ultimate penetration depth $D_{\max }$, as shown in Figure 14, and the relevant detailed influence extents are analysed in part 4.2. From the results in this figure, we can intuitively observe that as the ratio $r_{t} / a$ decreases, the penetration depth increases, whereas the penetration depth increases only slightly when $r_{t} / a$ is high (the penetration depth when $r_{t} / a=20$ is only $5.01 \%$ higher than that when $\left.r_{t} / a=60\right)$. At this time, the impact of the target size on the ultimate penetration depth is small, even negligible. However, when $r_{t} / a$ is low, especially when $r_{t} / a \leq 20$, the penetration depth suddenly increases greatly as $r_{t} / a$ decreases (the penetration depth when $r_{t} / a=2.5$ is $27.54 \%$ higher than that when $r_{t} / a=20$ ), and the impact of the target size on the ultimate penetration depth is increased, which is basically consistent with the theoretical calculation results.

4.2. Analysis of the Lateral Boundary Influence and Its Eliminating Condition. The theoretical and numerical results both show that when $r_{t} / a$ is small, there are substantial differences in the penetration performance between a rigid projectile penetrating into a thick, finite-radius, metallic target and that of the same projectile penetrating into an unlimited-size target. To analyse this difference further and quantify it, a large number of simulations of penetration under different working conditions when $r_{t} / a \leq 30$ and penetration of a semi-infinite target with the corresponding impact velocities are performed. Adding initial velocities in large scale to each model with different target radius-toprojectile radius ratios and comparing the penetration depth (D) results of the finite-radius aluminium-alloy targets with those of the semi-infinite aluminium-alloy targets, regular results based on sufficient simulation data are obtained, as shown in Figures 15-24. In these figures, the deviation value of the penetration depth is defined as the percentage of the difference between the penetration depth of the semi-infinite target and the penetration depth of the finite-radius target.

The penetration depth comparisons in Figures 15 and 16 indicate that the free lateral boundaries of the target have a substantial influence on the penetration damage when $r_{t} / a \leq 15$ and that the deviation in the penetration under these two boundary conditions can still reach $16.8 \%$ (far exceeding the given threshold of 5\%), although the projectile penetrates the target with a low initial velocity $(v=200 \mathrm{~m} / \mathrm{s})$. Thus, it can be considered that the effect of the free lateral boundaries of the target should not be ignored even if the striking velocity is low. Figures 17 and 18 show that, for $r_{t} / a=20$, the deviation in the penetration under these two boundary conditions is small when $v \leq 300 \mathrm{~m} / \mathrm{s}$; hence, the 


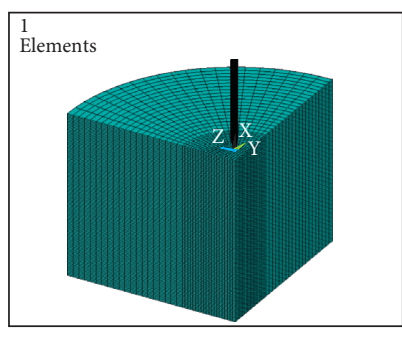

(a)

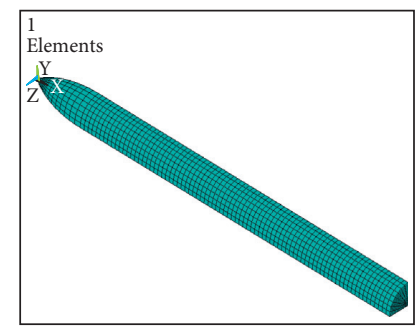

(b)

FIGURE 9: Meshing results of the finite element model: (a) global meshing and (b) projectile meshing.

TABle 3: Parameters of material models.

\begin{tabular}{|c|c|c|c|c|c|c|c|c|}
\hline Materials & $\rho\left(\mathrm{kgm}^{-3}\right)$ & $E(\mathrm{GPa})$ & $v$ & $A(\mathrm{MPa})$ & $B(\mathrm{MPa})$ & $n$ & $C$ & $m$ \\
\hline 4340 steel & 7830 & 200.6 & 0.29 & & & & & \\
\hline 6061-T6511 aluminum alloy & 2710 & 68.9 & $1 / 3$ & 192 & 218 & 0.14 & 0.0182 & 1 \\
\hline
\end{tabular}

Notes. $\rho$ is the density of the material, $E$ is the elasticity modulus of the material, $v$ is Poisson's ratio of the material, and $A, B, C, n, m$ are the model parameters: initial yield stress, hardening constant, strain rate constant, hardening exponent, and thermal softening exponent.

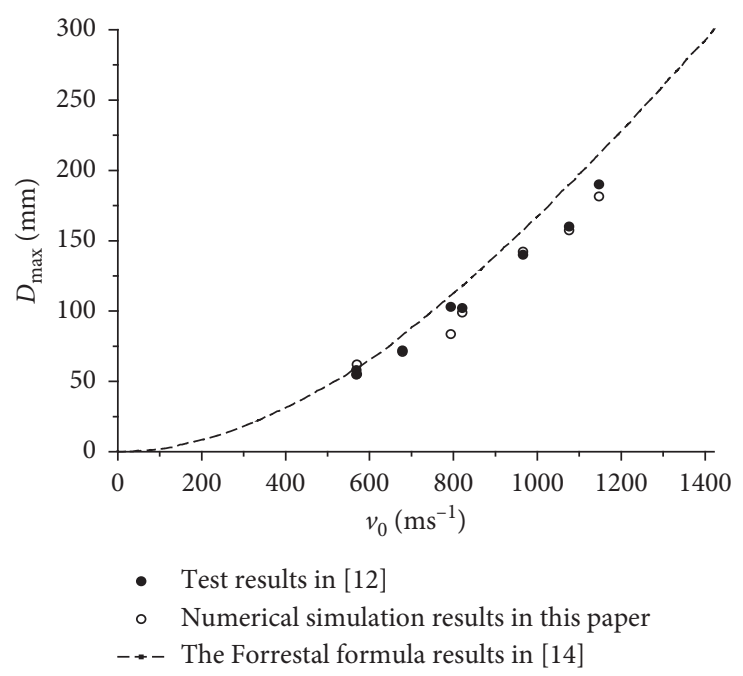

Figure 10: Comparison of penetration depth.

effect of the free lateral boundaries of the target on the penetration damage is slight $(\leq 5 \%)$. However, this effect increased significantly when $v=350 \mathrm{~m} / \mathrm{s}$, for which the deviation in the penetration is $9.2 \%$. Therefore, it can be considered that, for $r_{t} / a=20$, the effect of the free lateral boundaries of the target can be ignored when $v \leq 300 \mathrm{~m} / \mathrm{s}$. Similarly, for $r_{t} / a=25$, as shown in Figures 19 and 20, although the effect of the free lateral boundaries of the target on the penetration damage is slight $(\leq 5 \%)$ when $v=300 \mathrm{~m} / \mathrm{s}$, the deviation in the penetration can reach $20.5 \%$ when $v \leq 350 \mathrm{~m} / \mathrm{s}$; thus, for $r_{t} / a=25$, the effect of the free lateral boundaries of the target can be ignored when $v=300 \mathrm{~m} / \mathrm{s}$. Similarly, the plots in Figure 21 show that, for $r_{t} / a=26.5$, the effect of the target lateral free boundary on the penetration can be ignored when $v \leq 500 \mathrm{~m} / \mathrm{s}$ (the deviation in the penetration under these two boundary conditions is $5.2 \%$, which begins to slightly exceed the given threshold of 5\%). However, it can be seen from Figures 22-24 that, for $r_{t} / a \geq 28$, the effect of the free lateral boundaries of the target on the penetration damage is slight because the deviation in the penetration is small when $v \leq 700 \mathrm{~m} / \mathrm{s}$; this deviation is $2.1 \%$ when $r_{t} / a=28$ and $2.6 \%$ when $r_{t} / a=30(\leq 5 \%)$. 


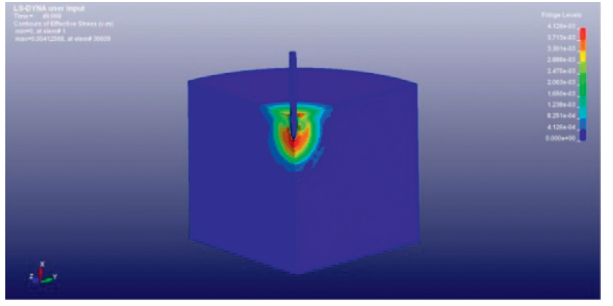

(a)

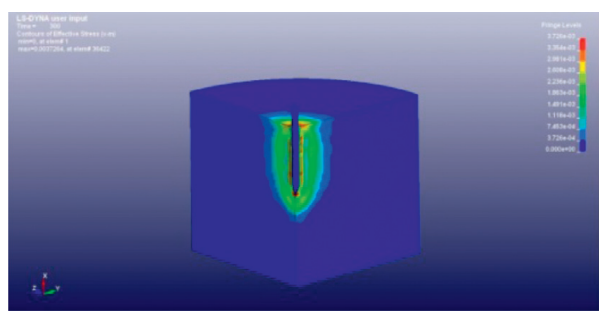

(c)

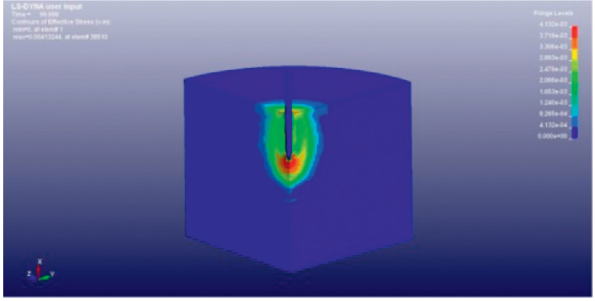

(b)

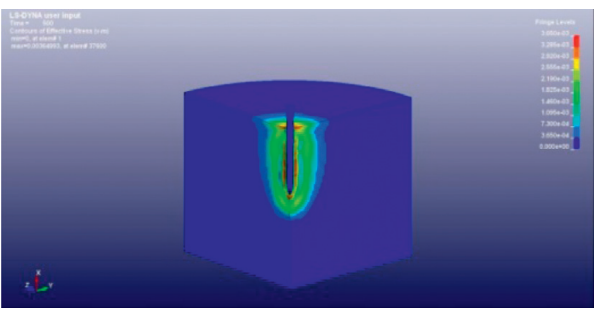

(d)

Figure 11: Stress nephograms of a projectile penetrating into a thick, finite-radius, metallic target at different times, (a) $t=0.05 \mathrm{~ms}$, (b) $t=0.1 \mathrm{~ms}$, (c) $t=0.3 \mathrm{~ms}$, and (d) $t=0.5 \mathrm{~ms}$.

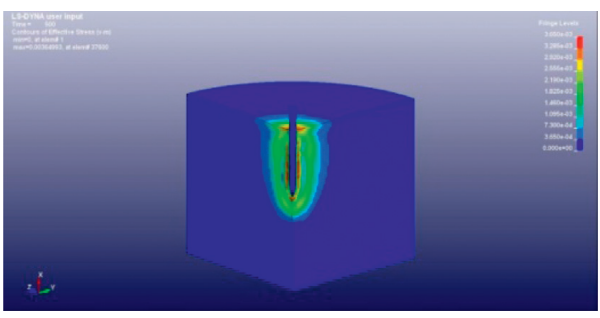

(a)

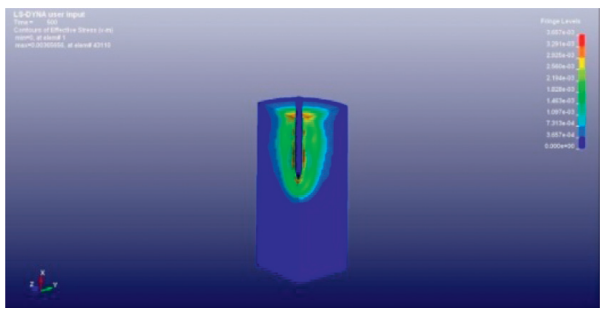

(c)

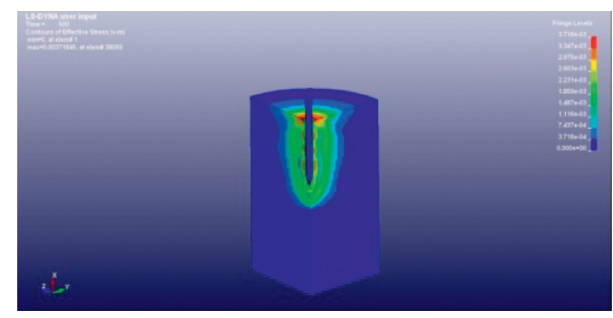

(b)

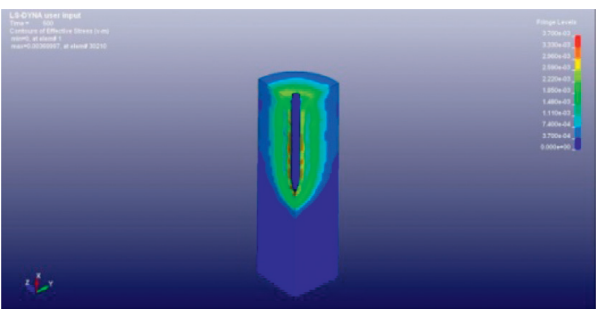

(d)

Figure 12: Final form of penetration models with different values of $r_{t} / a$, (a) $r_{t} / a=35.7$, (b) $r_{t} / a=20,(\mathrm{c}) r_{t} / a=15$, and (d) $r_{t} / a=10$.

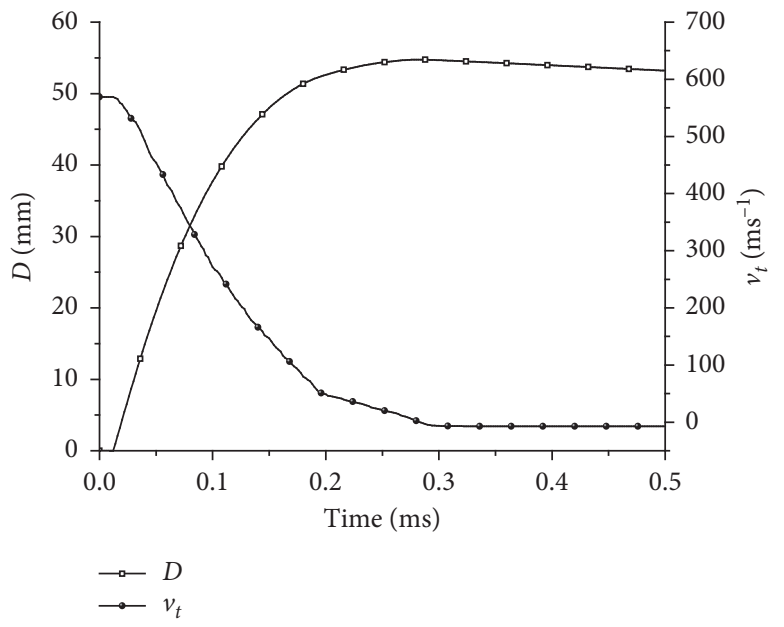

FIGURE 13: Time-history curves of the penetration depth and penetration velocity for the projectile. 


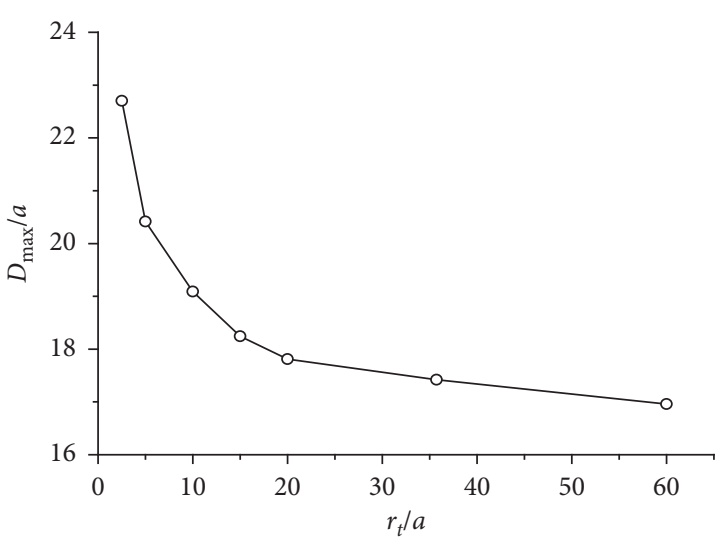

FIGURE 14: Influence curve of the target size on the penetration depth.

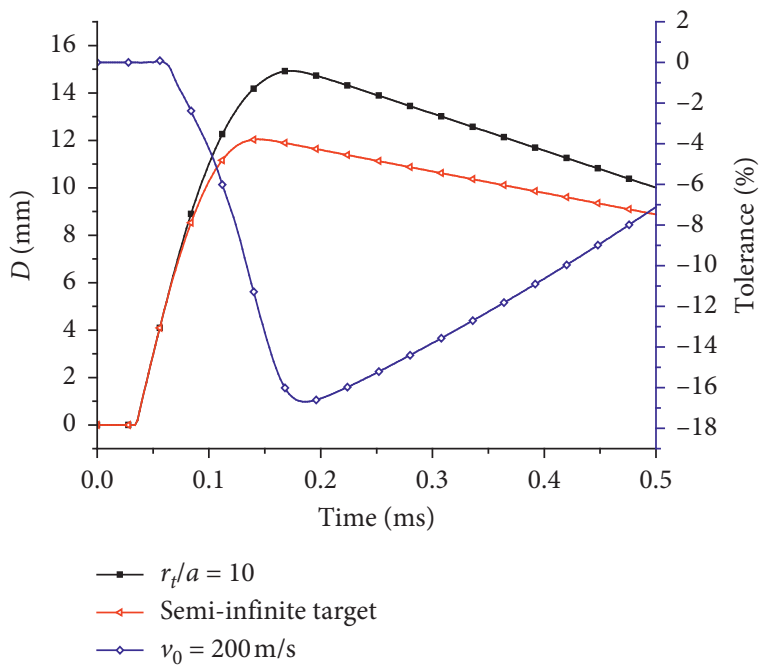

FIGURE 15: Deviation comparison of the penetration depth for the free boundary $\left(r_{t} / a=10\right)$ and the nonreflecting boundary $(v=200 \mathrm{~m} / \mathrm{s})$.

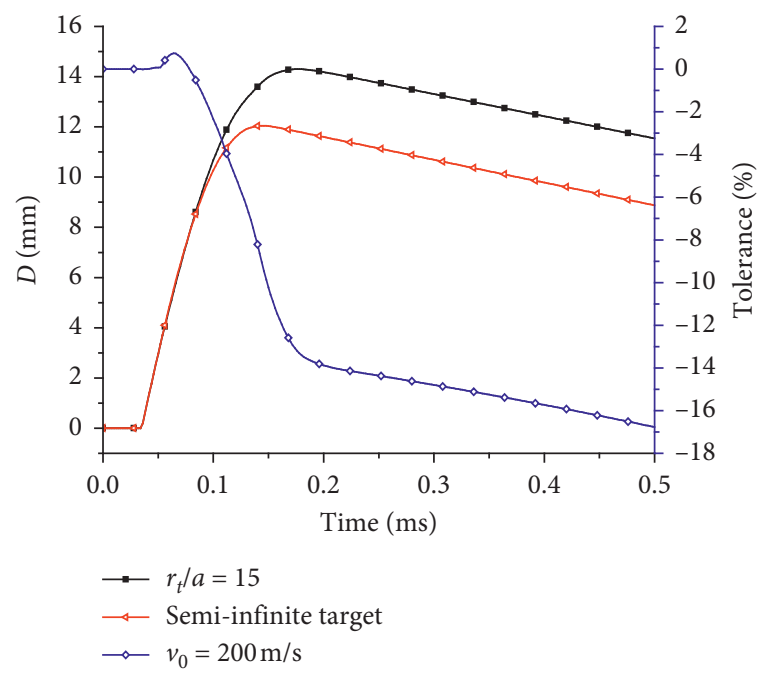

FIgURE 16: Deviation comparison of the penetration depth for the free boundary $\left(r_{t} / a=15\right)$ and the nonreflecting boundary $(v=200 \mathrm{~m} / \mathrm{s})$.

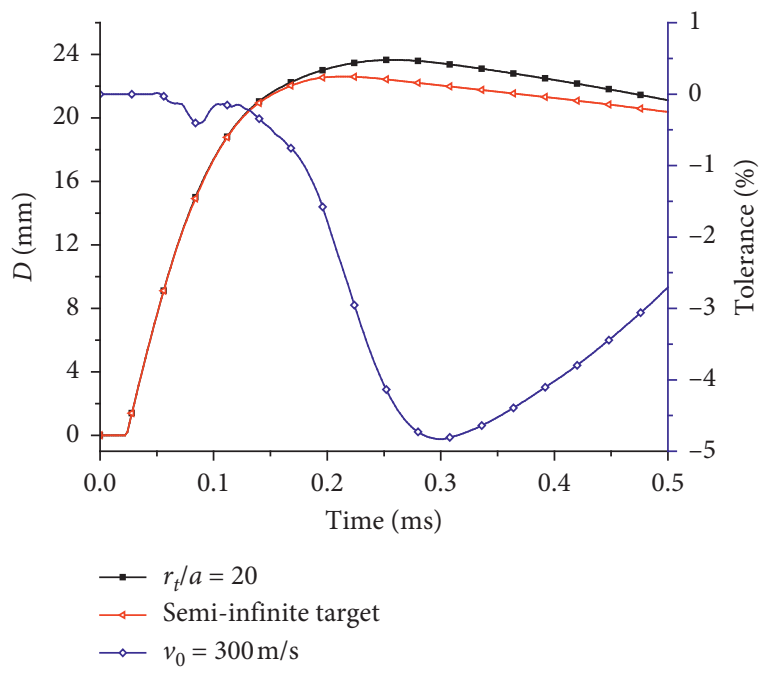

Figure 17: Deviation comparison of the penetration depth for the free boundary $\left(r_{t} / a=20\right)$ and the nonreflecting boundary $(v=300 \mathrm{~m} / \mathrm{s})$.

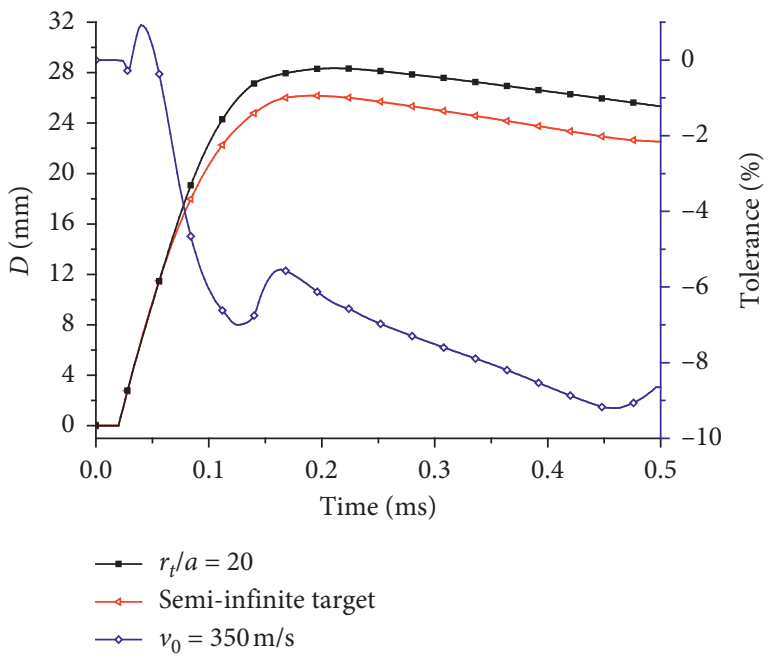

FIGURE 18: Deviation comparison of the penetration depth for the free boundary $\left(r_{t} / a=20\right)$ and the nonreflecting boundary $(v=350 \mathrm{~m} / \mathrm{s})$.

Consequently, the effect of the free lateral boundaries of the target on the penetration damage is considered to become insensitive when $r_{t} / a \geq 28$.

As the striking velocity is generally less than two Mach numbers in weapon site tests, combining the theoretical results and the numerical simulation results, for thick, finite-radius, metallic targets, the effect of the free lateral boundaries of the target on the penetration performance can be ignored by conservatively designing the target radius to be greater than 28 times the projectile radius. 


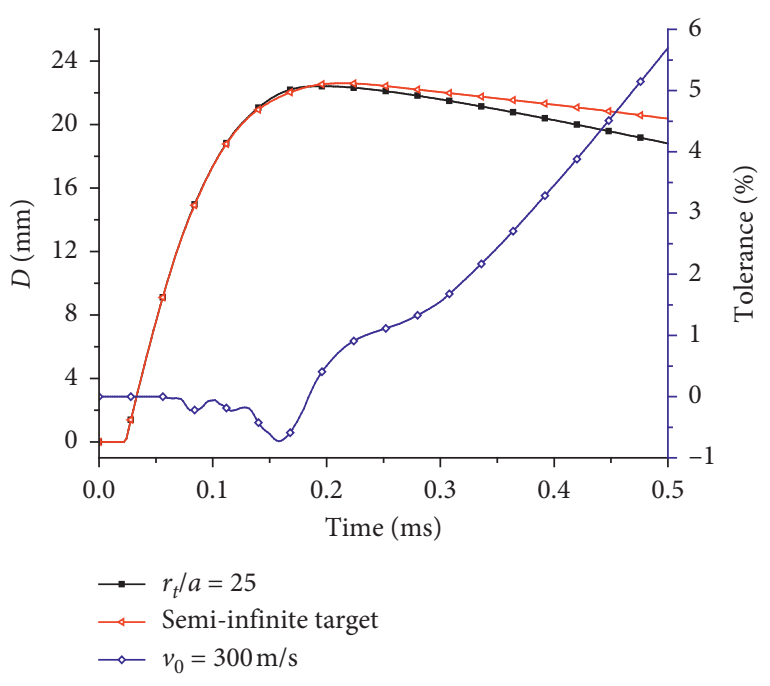

FIGURE 19: Deviation comparison of the penetration depth for the free boundary $\left(r_{t} / a=25\right)$ and the nonreflecting boundary $(v=300 \mathrm{~m} / \mathrm{s})$.

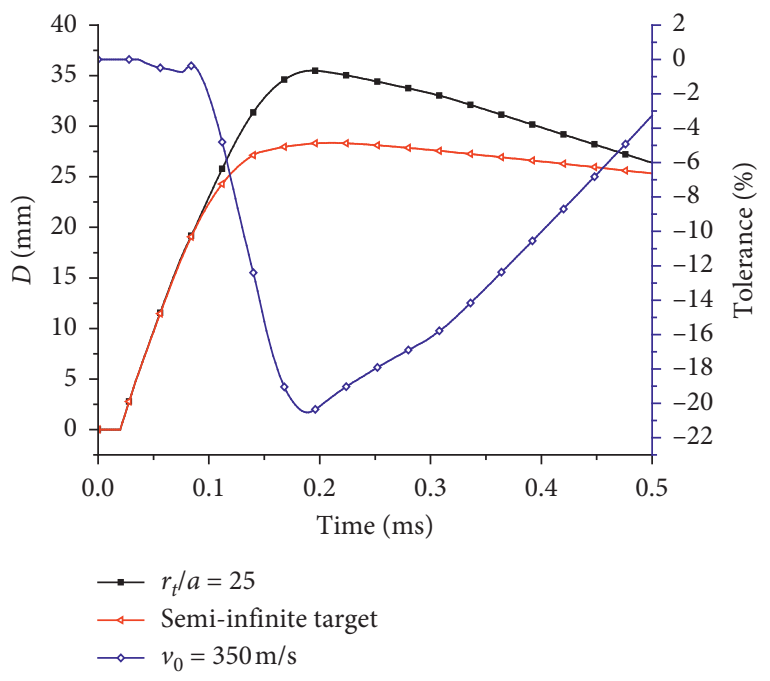

Figure 20: Deviation comparison of the penetration depth for the free boundary $\left(r_{t} / a=25\right)$ and the nonreflecting boundary $(v=350 \mathrm{~m} / \mathrm{s})$.

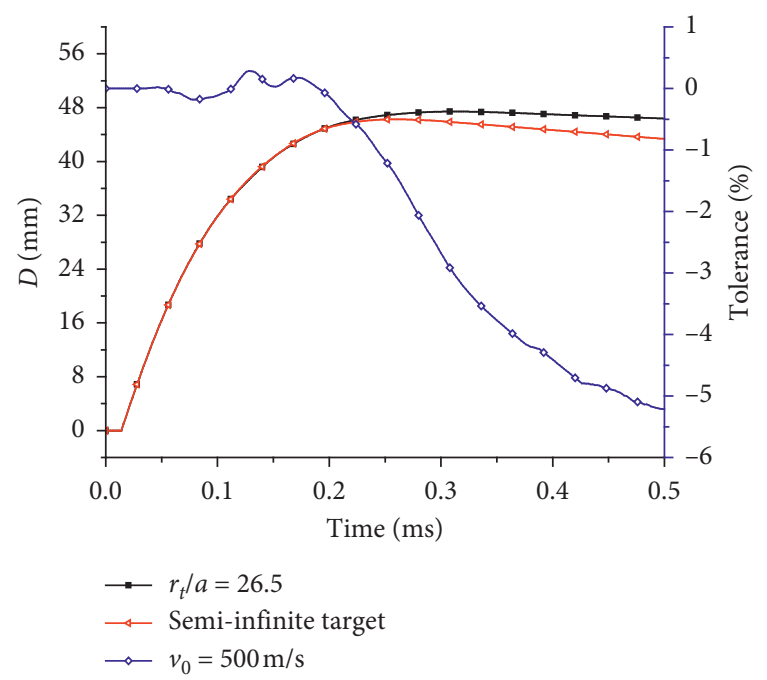

FIGURE 21: Deviation comparison of the penetration depth for the free boundary $\left(r_{t} / a=26.5\right)$ and the nonreflecting boundary $(v=500 \mathrm{~m} / \mathrm{s})$.

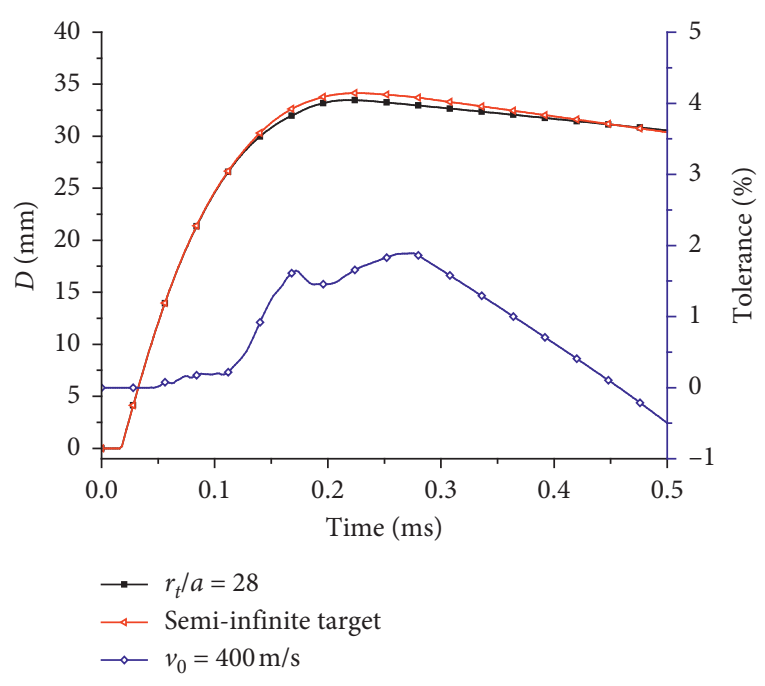

FIGURE 22: Deviation comparison of the penetration depth for the free boundary $\left(r_{t} / a=28\right)$ and the nonreflecting boundary $(v=400 \mathrm{~m} / \mathrm{s})$.

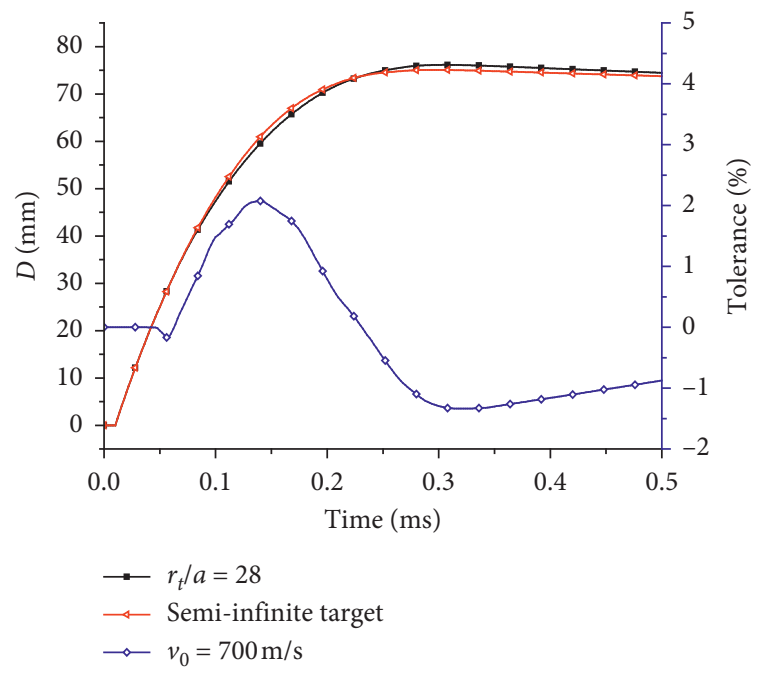

FIGURE 23: Deviation comparison of the penetration depth for the free boundary $\left(r_{t} / a=28\right)$ and the nonreflecting boundary $(v=700 \mathrm{~m} / \mathrm{s})$.

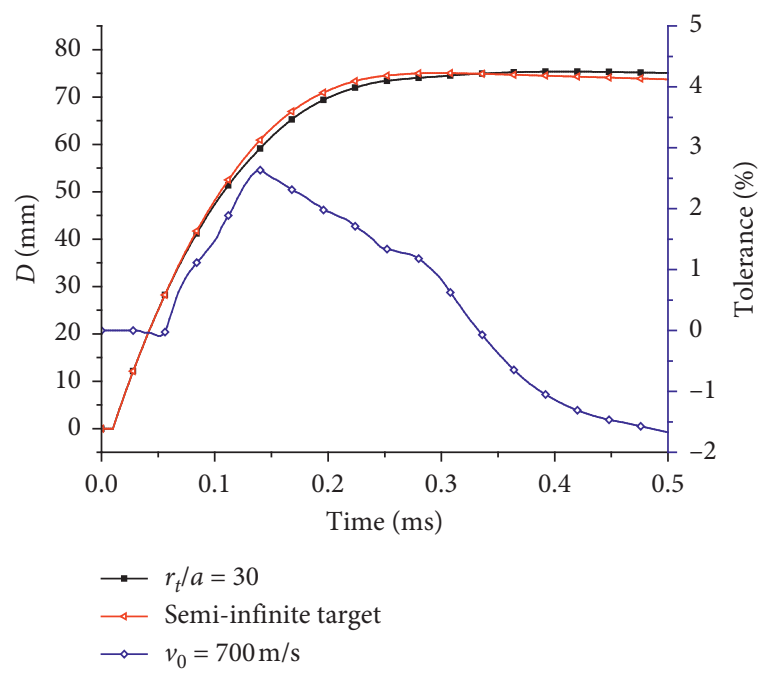

FIgURE 24: Deviation comparison of the penetration depth for the free boundary $\left(r_{t} / a=30\right)$ and the nonreflecting boundary $(v=700 \mathrm{~m} / \mathrm{s})$. 


\section{Conclusions}

(1) Considering the effect of the free lateral boundaries of the target, the intermediate principal stress, and the different strength criteria, the finite cylindrical cavity expansion model based on the unified strength theory was built in this paper. Then, penetration resistance formulas and penetration depth formulas for rigid projectiles penetrating into thick, finite-radius, metallic targets were deduced, which can be applied to resolve the complicated stress problems of all kinds of targets with equal tensile strength and compressive strength and different nose shapes. Additionally, these formulas are suitable for penetration problems of semi-infinite metallic targets. According to a comparative analysis, the calculation results in this paper are in better agreement with the experimental results (i.e., have higher precision) than the calculation results in relevant documents.

(2) The calculation method in this paper can obtain a series of analytical solutions based on different strength criteria to predict penetration depth ranges of all kinds of targets with equal tensile strength and compressive strength. The results in [14] are only a special case of the results deduced in this paper when $b=0.366$. The strength parameter $b$ had an obvious influence on the penetration results. It is considering the intermediate principal stress effect that can objectively show the strength potential of the material and ensure that the antipenetration performance of the member is fully utilized.

(3) When $r_{t} / a \leq 10$, the finite cylindrical cavity expansion model fully undergoes the elastoplastic stage and the plastic stage, whereas when $r_{t} / a>10$, it only undergoes the elastoplastic stage. As $r_{t} / a$ decreases, $D_{\max } / a$ increases, and the extent of this increase is obvious when $r_{t} / a<16$. The results showed that the penetration depth was obviously affected by the target boundary size under these conditions, and the calculations cannot be performed under the assumption of an unlimited-size target.

(4) According to the combined theoretical analysis and numerical simulation results, if the target radius is designed to be greater than 28 times the projectile radius, the effect of the free lateral boundaries of the target on the penetration performance can be ignored in weapons field tests. Furthermore, the results showed that the striking velocity of the projectile and the shape of the projectile nose also greatly influenced the penetration depth.

\section{Data Availability}

The data used to support the findings of this study are available from the corresponding author upon request.

\section{Conflicts of Interest}

The authors declare that there are no conflicts of interest regarding the publication of this paper.

\section{Acknowledgments}

This study was financially supported by the National Natural Science Foundation of China (Grant no. 51878056), the Social Development Foundation for Science and Technology Planning Project of Shaanxi Province (Grant no. 2019SF256), and the Fundamental Research Funds for the Central Universities, CHD (Grant nos. 300102289105 and 300102280203).

\section{References}

[1] M. J. Forrestal and T. L. Warren, "Perforation equations for conical and ogival nose rigid projectiles into aluminum target plates," International Journal of Impact Engineering, vol. 36, no. 2, pp. 220-225, 2009.

[2] X. Y. Zhang, H. J. Wu, S. Zhang, and F. L. Huang, "Projectile penetration of reinforced concrete considering the effect of steel reinforcement: experimental study and theoretical analysis," International Journal of Impact Engineering, vol. 144, Article ID 103653, 2020.

[3] Y. H. Yoo, J. B. Kim, and C. W. Lee, "Effects of the projectile geometries on normal and oblique penetration using the finite cavity pressure method," Applied Sciences, vol. 9, no. 18, Article ID 9183939, 2019.

[4] C. Liu, X. F. Zhang, C. Q. Huang et al., "Critical velocity theoretical model for hemispherical long rod projectiles' penetrating semi-infinite thick target at high velocity," Journal of Vibration and Shock, vol. 38, no. 9, pp. 8-14, 2019.

[5] M. Zhen, Z.-G. Jiang, D.-Y. Song, and F. Liu, "Analytical solutions for finite cylindrical dynamic cavity expansion in compressible elastic-plastic materials," Applied Mathematics and Mechanics, vol. 35, no. 8, pp. 1039-1050, 2014.

[6] Y. Liu, A. G. Pi, H. Yang et al., "Study on similarity law of nonproportionally scaled penetration/perforation test," Explosion and Shock Waves, vol. 40, no. 3, pp. 63-75, 2020.

[7] X. W. Chen, X. L. Li, F. L. Huang, H. J. Wu, and Y. Z. Chen, "Damping function in the penetration/perforation struck by rigid projectiles," International Journal of Impact Engineering, vol. 35, no. 11, pp. 1314-1325, 2008.

[8] Y. Li, W. Fan, J. H. Zhao et al., "Dynamic response study for penetration of medium-low speed projectile on semi-infinite rock targets," Engineering Mechanics, vol. 34, no. 9, pp. 139-149, 2017.

[9] Y. B. Wang, M. H. Yu, and J. D. Lin, "Cylindrical cavity expansion model for projectile normally penetrating into concrete target," Journal of Xi'an Jiaotong University, vol. 38, no. 3, pp. 303-307, 2004.

[10] Y.-B. Wang, X.-Q. Zhang, M.-H. Yu, and J.-D. Lin, "Penetration analysis of long rod projectile on rock targets," Chinese Journal of Rock Mechanics and Engineering, vol. 24, no. 8, pp. 1301-1307, 2005.

[11] D. L. Littlefield, C. E. Anderson, Y. Partom, and S. J. Bless, "The penetration of steel targets finite in radial extent," International Journal of Impact Engineering, vol. 19, no. 1, pp. 49-62, 1997.

[12] A. J. Piekutowski, M. J. Forrestal, K. L. Poormon, and T. L. Warren, "Penetration of 6061-T6511 aluminum targets 
by ogive-nose steel projectiles with striking velocities between 0.5 and $3.0 \mathrm{~km} / \mathrm{s}$," International Journal of Impact Engineering, vol. 23, no. 1, pp. 723-734, 1999.

[13] Z. G. Jiang, D.-Y. Song, and S.-Y. Zeng, "A finite cylindrical cavity expansion theory and its application," Journal of $\mathrm{Vi}$ bration and Shock, vol. 30, no. 4, pp. 139-143, 2011.

[14] D. Y. Song, F. Liu, and Z. G. Jiang, "An analytical model for penetration into cylindrical metallic thick target by rigid sharp-nosed projectiles," Engineering Mechanics, vol. 30, no. 1 , pp. 31-36, 2013

[15] S. S. Sun, J. H. Zhao, and C. G. Zhang, "Calculation of silo wall pressure considering the intermediate stress effect," Advances in Civil Engineering, vol. 2018, Article ID 3673515, 10 pages, 2018.

[16] C.-G. Zhang, X.-D. Chen, and W. Fan, "Critical embedment depth of a rigid retaining wall against overturning in unsaturated soils considering intermediate principal stress and strength nonlinearity," Journal of Central South University, vol. 23, no. 4, pp. 944-954, 2016.

[17] M. H. Yu, New System of Strength Theory, Xi'an: Xi'an Jiaotong University Press, Xi'an, China, 1992.

[18] J. H. Zhao, Unified Strength Theory and its Engineering Application, Science Press, Beijing, China, 2003.

[19] R. Hill, The Mathematical Theory of Plasticity, Oxford University Press, London, UK, 1950.

[20] C.-W. Jin, L.-Z. Wang, and Y.-Q. Zhang, "Strength differential effect and influence of strength criterion on burst pressure of thin-walled pipelines," Applied Mathematics and Mechanics, vol. 33, no. 11, pp. 1361-1370, 2012.

[21] Z. G. Jiang, S.-Y. Zeng, and J.-P. Zhou, "A three-stage model for the perforation of moderately thick metallic plates," Acta Armamentarii, vol. 28, no. 9, pp. 1046-1052, 2007.

[22] H. H. Zou, C. M. Song, M. Y. Wang, D. R. Wang, and D. S. Wen, "Experimental and numerical simulation study on the antipenetration properties of fiber ceramic-reactive powder concrete composite targets," Shock And Vibration, vol. 2019, Article ID 7673437, 16 pages, 2019. 